\title{
Single Nucleotide Polymorphisms and Haplotypes in Vascular Endothelial Growth Factor Gene and Residual Ridge Resorption of Mandible in Korean Population
}

\author{
Jung-Hyun Song, Jae-Hoon Lee ${ }^{*}$
}

Department of Prosthodontics, College of Dentistry, Yonsei University, Seoul, Korea.

Email: *jaehoon115@yuhs.ac

Received October $29^{\text {th }}, 2013$; revised December $16^{\text {th }}, 2013$; accepted January $5^{\text {th }}, 2014$

Copyright (C) 2014 Jung-Hyun Song, Jae-Hoon Lee. This is an open access article distributed under the Creative Commons Attribution License, which permits unrestricted use, distribution, and reproduction in any medium, provided the original work is properly cited. In accordance of the Creative Commons Attribution License all Copyrights (C) 2014 are reserved for SCIRP and the owner of the intellectual property Jung-Hyun Song, Jae-Hoon Lee. All Copyright (C) 2014 are guarded by law and by SCIRP as a guardian.

\begin{abstract}
The tooth extraction is a routine surgical procedure in the dental treatment where the healing process results in a saddle-shaped residual ridge in the edentulous jaw. There are substantial differences among individuals in the end result. In some cases, there is excessive bone atrophy, which complicates the dental restorative treatment. The alveolar ridge receives the mechanical load continuously from the periodontal ligament connected to the teeth and it diminishes dramatically as a consequence of dental extraction; thus it is believed that the continuing pattern of the alveolar bone resorption is related to this change. The reduced partial pressure of oxygen is the most prominent event from the reduced mechanical load. Vascular Endothelial Growth Factor (VEGF), regulated by HIF-1, reported close association with angiogenesis and bone turn over, where partial oxygen pressure has changed. Therefore the genetic association between Single Nucleotide Polymorphsim (SNP) of VEGF gene and RRR was investigated. 120 subjects $(70.93 \pm 9.28$ years) which were treated at Dental clinic of Yonsei University with edentulous mandible were recruited. Mandibular bone height was measured following the protocol of the American College of Prosthodontists. Three variants, rs1570360, rs25648, and rs3025039 in VEGF from previous study, were used as tag-SNPs and genotyping for the study. Student's t-test and ANOVA were used for statistical analysis. There was a notable association with rs1570360 $(P=0.051)$ in dominant group and haplotype A-C-C showed a statistically significant association with RRR in dominant group $(P=\mathbf{0 . 0 4 2})$. Results of this study may be useful in developing novel genetic diagnostic tests and identifying Koreans susceptible to developing severe RRR after dental extraction.
\end{abstract}

\section{KEYWORDS}

SNP; VEGF; Haplotype; Residual Ridge Resorption; Edentulous Mandible

\section{Introduction}

The tooth extraction is one of the most frequently performed dental procedures. After the tooth extraction, the residual ridge undergoes the process of the continual bone resorption due to the reduced mechanical loading of the tooth and the dental ligament [1]. With the increase of the life expectancy of the elderly population, edentulous patients are increasing as well. The results and satisfactions from complete denture treatments, commonly used for

${ }^{*}$ Corresponding author. edentulous patients, are dependent upon the preservation of the residual ridge. During the denture use, the degree of the residual ridge resorption influences the masticatory and the nutrient intake ability. This greatly affects the quality of life and the life expectancy. Therefore, as a part of the research on the prosthodontic treatment, studies on the residual ridge resorption are necessary to give patients more functional satisfaction.

The residual ridge resorption after the tooth extraction has been studied extensively in the past [2-5]. After the tooth extraction, the patient undergoes the loss of alveo- 
lar process and is viable to mucosa-borne prosthesis. This represents unphysiological occlusal loading which brings reduced masticatory function and lack of forces into the bone [5]. Then mineral content in the bone rapidly decreases which leads to the bone resorption [6]. Several internal and external factors are involved in the study; however, research on genetic factor has been overlooked.

Once tooth is extracted, blood vessel in the extraction socket gets injured. Then blood supply is blocked and the oxygen pressure of the surrounding area decreases, which makes a state of hypoxia within the tissue [7]. Our body tries to maintain homeostasis by expanding blood vessels, increasing vascular development and angiogenesis. Angiogenesis is the most effective and long-term adaptive response. During the process of homeostasis, osteocyte works as a biological signal regulating several other mediators. Among such mediators, HIF-1 is the master regulator of genes dependent on oxygen concentration. HIF-1 is closely involved in the bone remodeling and the osteoclastic bone resorption. Of the 60 or more genes that are expressed by HIF-1, VEGF is involved in angiogenesis and is one of the target genes of the bone remodeling [8]. It has been reported that VEGF is quickly upregulated once HIF-1 expression increases [9]. VEGF is also important for the osteoclastic bone resorption and acts as a vital factor for the normal bone remodeling, while the new endothelial vessel expressed by VEGF is an essential step for the bone deposition where the bone resorption occurs by osteoclast. Therefore it can be hypothesised that genetic variation in VEGF might affect the residual ridge resorption in mandible.

\section{Material and Method}

\subsection{Ethics Statement}

All research involving human subjects or human data was approved by the Institutional Review Board of Yonsei University College of Dentistry (Yonsei IRB No. 22010-0022). All clinical investigation was performed in accordance with the Declaration of Helsinki. Written informed consent was obtained from all participants before taking part in this study [10].

\subsection{Study Population}

The study population consisted of 120 unrelated Korean individuals, 41 men and 79 women who were recruited between January 2011 and February 2013 from Yonsei University Dental Hospital. The men were aged (mean $71.12 \pm 8.41$ years) and the women were aged between (mean $70.21 \pm 9.75$ years). Each subject was completely or partially edentulous for at least two years. The partially edentulous subjects were missing both maxillary and mandibular premolars and molars either in unilateral or bilateral sides. Those subjects that met the following criteria were included in the study: 1) no known systemic conditions that could affect bone conditions such as osteoporosis, other metabolic bone disease, or pituitary disease; 2) no history of bone transplantation; and 3) presenting a panoramic dental radiograph less than 2 years old. After informed consent was obtained, an oral examination was performed to confirm their edentulous condition [10].

\subsection{Measurement of Mandibular Residual Ridge Height}

The panoramic dental radiograph of each subject was digitized with an anonymously assigned identification number and stored in a secured computer with password protection. Following the Prosthodontic Diagnosis Index from the American College of Prosthodontists, the lowest height of the edentulous mandible was measured (Figure 1). The mean of mandibular bone height was $15.03 \pm 4.07$ $\mathrm{mm}(\mathrm{n}=120)$ and varied from $6.55 \mathrm{~mm}$ to $25.73 \mathrm{~mm}$.

\subsection{Genomic DNA Extraction}

Each edentulous subject was asked to collect $2 \mathrm{ml}$ of saliva in the tube of an Oragene DNA Self-Collection Kit that contained $2 \mathrm{ml}$ of DNA-preserving solution (DNA
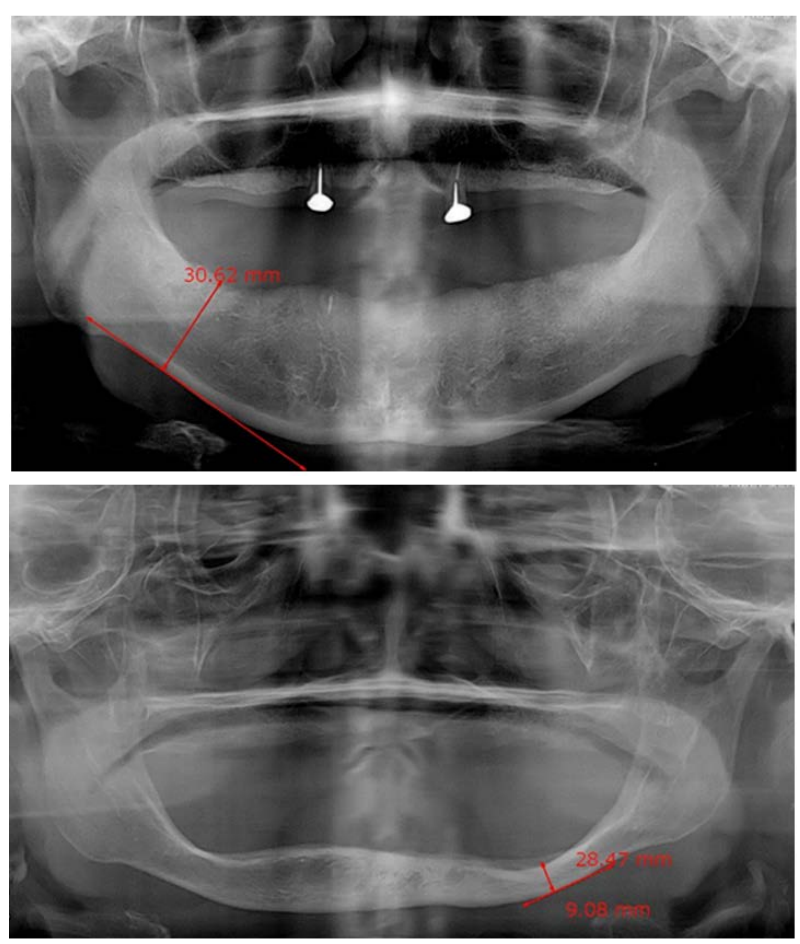

Figure 1. Characterization of mandibular ridge. The lowest residual height (arrow) of the edentulous mandible was determined by panoramic dental radiographs. The figure on the right shows severe atrophy of the edentulous mandible. 
Genotek Cat. \#OG-250). When the saliva was collected, the lid was closed and the liquid in the lid was released into the tube to mix with the saliva. The sample with a total volume of $4 \mathrm{ml}$ was stored at $25^{\circ} \mathrm{C}$ for no longer than 2.5 weeks. Genomic DNA was collected using the Puregene DNA purification kit from Qiagen (Valencia, CA, USA) according to the manufacturer's protocol. DNA extractions and further analysis were done by DNA Link Inc. (Seoul, South Korea). The concentration of DNA was measured with a Nanodrop Spectrophotometer-1000 (Thermo Scientific, Delaware, USA) [10].

\subsection{Use of Tag SNPs}

Seven SNPs of VEGF gene were already identified in previous study [1]. Therefore whole sequencing was not necessary for the study. Three out of seven SNPs were selected based on allele frequency and linkage disequilibrium (LD) and were used as tag SNP in this study (Figure 2). Three tag SNPs that were used are following: rs1570360 in chr6:43737830, rs25648 in chr6:43738977, rs3025039 in chr6:43752536.

\subsection{Statistical Analysis}

All data analysis was completed using the statistics software SAS 9.1.3 (SAS Institute Inc. in Cary, NC, US). Hardy-Weinberg equilibrium (HWE) tests were evaluated to ensure genotyping quality. Three variants, rs1570360, rs25648, and rs3025039 in VEGF from previous study were used as tag-SNPs and genotyping for the study [1].

The association of SNPs and haplotypes, frequency rates over $5 \%$, with the mandibular jawbone atrophy was evaluated using Student's t-test for dominant and recessive groups, and ANOVA for the codominant group. Moreover, statistical analysis was performed to find the relationship between the ages, the time since tooth extraction of the patients, and the height of mandible. Linear Regression was used to verify the independence among the three factors [10].

\section{Result}

Measured mean height of mandibular bone height was $15.30 \mathrm{~mm}$. Frequencies and association of three tag SNPs are listed on Table 1 and 2. Three individual tag SNPs did not show statistically significant association with RRR. rs 1570360 showed $p$-value of 0.051 , which is very near to 0.05 . Other two SNPs did not show any association. On the other hand, Haplotype A-C-C, the second most prevalent type showed association with RRR (Tables 3 and 4, Figure 3, $P$ value $=0.042$ ).

\section{Discussion}

The bone remodeling is continuous throughout life and is

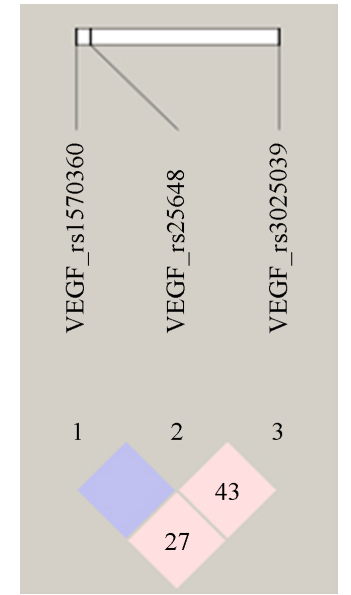

Figure 2. Haplotypes of VEGF gene. Three variants, rs1570360, rs25648, and rs3025039 in VEGF were used as tag-SNPs and genotyping.
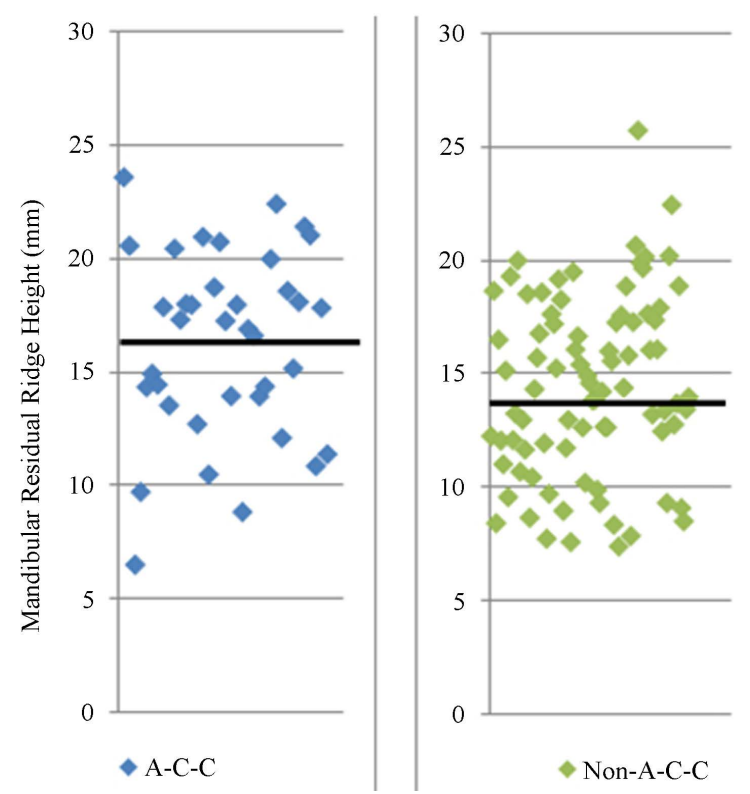

Figure 3. Scatter plot of mandibular residual ridge height of total patients $(n=120)$ divided into two groups by haplotype A-C-C and non A-C-C. Bold lines across the dots indicate the average of measured mandibular residual ridge height.

an essential part of normal physiology and homeostasis to adapt to new circumstances. In the homeostasis point, the body will regulate the deposition and the resorption of bone as necessary, which may be controlled by the angiogenesis gene such as HIF (Hypoxia inducible factor) and VEGF (Vascular Endothelial Growth Factor). After the tooth extraction, bone tissues in residual ridge become hypoxic by the reduced mechanical loading which is delivered by periodontal ligament. Bone tissues in the residual ridge face a new situation that requires a glycollytic ATP generation due to hypoxia, which is very 
Table 1. Frequencies of VEGF polymorphisms in 120 Koreans.

\begin{tabular}{|c|c|c|c|c|c|c|c|c|}
\hline Position & SNP number & & & & & MAF & Heterozygosity & HWE \\
\hline \multirow[b]{2}{*}{ Chr6: 43737830} & \multirow[b]{2}{*}{ rs1570360 } & GG & GA & AA & $\mathrm{N}$ & \multirow[b]{2}{*}{0.175} & \multirow[b]{2}{*}{0.3} & \multirow[b]{2}{*}{1} \\
\hline & & 81 & 36 & 3 & 120 & & & \\
\hline \multirow{2}{*}{ Chr6: 43738977} & \multirow{2}{*}{ rs25648 } & CC & CT & TT & $\mathrm{N}$ & \multirow{2}{*}{0.063} & \multirow{2}{*}{0.108} & \multirow{2}{*}{0.370} \\
\hline & & 106 & 13 & 1 & 120 & & & \\
\hline \multirow{2}{*}{ Chr6: 43752536} & \multirow{2}{*}{ rs3025039 } & CC & CT & TT & $\mathrm{N}$ & \multirow{2}{*}{0.154} & \multirow{2}{*}{0.273} & \multirow{2}{*}{0.727} \\
\hline & & 85 & 33 & 2 & 120 & & & \\
\hline
\end{tabular}

Table 2. Association of VEGF polymorphisms in 120 Koreans.

\begin{tabular}{ccccc}
\hline Loci & $\begin{array}{c}\text { SNP } \\
\text { number }\end{array}$ & $\begin{array}{c}\text { Dominant } \\
P \text { value }\end{array}$ & $\begin{array}{c}\text { Recessive } \\
P \text { value }\end{array}$ & $\begin{array}{c}\text { Codominant } \\
P \text { value }\end{array}$ \\
\hline chr6:43737830 & rs1570360 & 0.051 & 0.688 & 0.15 \\
chr6:43738977 & rs25648 & 0.767 & 0.293 & 0.576 \\
chr6:43752536 & rs3025039 & 0.644 & 0.766 & 0.88 \\
\hline
\end{tabular}

Table 3. Association of VEGF haplotypes in 120 Koreans.

\begin{tabular}{ccccc}
\hline Haplotype & Frequency & $\begin{array}{c}\text { Dominant } \\
P \text { value }\end{array}$ & $\begin{array}{c}\text { Recessive } \\
P \text { value }\end{array}$ & $\begin{array}{c}\text { Codominant } \\
P \text { value }\end{array}$ \\
\hline G-C-C & 0.678 & 0.485 & 0.092 & 0.678 \\
A-C-C & 0.111 & $0.042^{*}$ & 0.95 & 0.111 \\
A-C-T & 0.069 & 0.636 & - & 0.069 \\
\hline
\end{tabular}

Table 4. Haplotype A-C-C showed less atrophied jawbone level than others.

\begin{tabular}{cccccc}
\hline Model & $P$-value & Genotype & N & Mean & Std \\
\hline \multirow{2}{*}{ Dominant } & 0.041693 & A-C-C & 23 & 20.727 & 5.17 \\
& & Other & 97 & 17.857 & 6.187 \\
\multirow{2}{*}{ Recessive } & 0.950019 & A-C-C & 1 & 18.79 & \\
& & Other & 119 & 18.404 & 6.117 \\
Codominant & 0.120174 & A-C-C Other & 22 & 20.815 & 5.274 \\
& & A-C-C A-C-C & 1 & 18.79 & \\
& & Other & 97 & 17.857 & 6.187 \\
\hline
\end{tabular}

$P$ value $<0.05$.

expensive from an energetic viewpoint. Therefore new blood vessel formation is inevitable to increase the oxygen delivery to the bone tissue. HIF is the fundamental hypoxia-response protein and over 70 genes have been identified to be HIF-dependent. Among those genes, VEGF-induced angiogenesis is one of the several keys to hypoxia adaptation. Such process increases oxygen deli- very by boosting angiogenesis. Angiogenesis and bone remodeling are closely linked. VEGF not only increases oxygen pressure in the bone tissue but also directs autocrine in osteoclast differentiation. VEGF is a proangiogenic cytokine that has been shown to promote osteoclast differentiation and survival. Studies have also revealed that VEGF acts as a macrophage-colony stimulating factor (M-CSF) during osteoclastogenesis in the osteopetrotic mouse model. By enhancing osteoblast and osteoclast activity in the bone remodeling process of the residual ridge, VEGF might be the regulating gene consistently increasing bone resorption in order to adapt to hypoxic circumstances, which will eventually reduce the consumption of unnecessary oxygen. Therefore it is possible to assume that genetic variation in VEGF directly or indirectly affects level in residual ridge resorption.

VEGF gene is highly polymorphic; hundreds of polymorphisms are annotated in dbSNP. These SNPs are grouped in three major linkage disequilibrium (LD) blocks [11]. The first one is located upstream of the transcription start point; the second one includes the promoter and the first six exons; the third one covers the last two exons and the 3' UTR [12]. Only a small number of reports are available concerning SNPs on the first block. The second block, however, consists of well known SNPs associated with many diseases. rs1570360 and rs25648 are typical SNPs in the promoter region and are often found from direct sequencing of the Korean population. rs1570360, showed remarkable association in this study, may also be representative of other well known SNPs in the promoter region as it shows strong LD with rs699947 and rs2010963. 3' UTR of the well known polymorphism rs3025039 should be tested to discriminate the major genotypes that are located in the third block. Therefore the three SNPs studied in this research cover the major functional SNPs of recent VEGF studies.

Although each of the three SNPs did not show any statistically significant association, haplotype A-C-C from this study, which is the second prevalent type containing the 2nd and the 3rd LD block, showed association with 
the residual ridge resorption. The haplotype generally presents more strong association evidence than individual SNP genotype. Because the effect of genotype on VEGF expression is cell-type and stimulus specific [11], this seems to be a well adaptive process in the physiological point of view since residual ridge lost its function to support the tooth and advanced to the process of atrophy. Other haplotypes might be less functional to this degeneration.

\section{Acknowledgements}

This work was supported by the National Research Foundation of Korea (NRF) funded by the Ministry of Education, Science and Technology (7-2011-0259).

\section{REFERENCES}

[1] Y. Kim, Y. J. Nam and C. Lee, "Haplotype Analysis of Single Nucleotide Polymorphisms in VEGF Gene for Vascular Dementia," American Journal of Medical Genetics Part B: Neuropsychiatric Genetics, Vol. 141B, No. 4, 2006, pp. 332-335. http://dx.doi.org/10.1002/ajmg.b.30317

[2] H. Devlin and M. W. Ferguson, “Alveolar Ridge Resorption and Mandibular Atrophy. A Review of the Role of Local and Systemic Factors,” British Dental Journal, Vol. 170, No. 3, 1991, pp. 101-104. http://dx.doi.org/10.1038/sj.bdj.4807427

[3] T. Haraldson, U. L. F. Karlsson and G. E. Carlsson, "Bite Force and Oral Function in Complete Denture Wearers," Journal of Oral Rehabilitation, Vol. 6, No. 1, 1979, pp. 41-48.

http://dx.doi.org/10.1111/j.1365-2842.1979.tb00403.x

[4] A. Tallgren, "The Continuing Reduction of the Residual Alveolar Ridges in Complete Denture Wearers: A MixedLongitudinal Study Covering 25 Years," The Journal of Prosthetic Dentistry, Vol. 89, No. 5, 2003, pp. 427-435.
http://dx.doi.org/10.1016/S0022-3913(03)00158-6

[5] A. Tallgren, B. R. Lang, G. F. Walker and M. M. Ash, "Roentgen Cephalometric Analysis of Ridge Resorption and Changes in Jaw and Occlusal Relationships in Immediate Complete Denture Wearers," Journal of Oral Rehabilitation, Vol. 7, No. 1, 1980, pp. 77-94. http://dx.doi.org/10.1111/j.1365-2842.1980.tb01466.x

[6] S. Hansson and A. Halldin, "Alveolar Ridge Resorption after Tooth Extraction: A Consequence of a Fundamental Principle of Bone Physiology," Journal of Dental Biomechanics, Vol. 3, 2012.

[7] J.-J. Kim, H.-C. Shon, J.-S. Chang, J.-H. Kim, K.-S. Lee and S.-W. Kim, "HIF-1 Alpha and VEGF Expression in Fracture Healing," The Journal of the Korean Orthopaedic Association, Vol. 43, No. 4, 2008, pp. 479-487. http://dx.doi.org/10.4055/jkoa.2008.43.4.479

[8] J. W. Lee, S. H. Bae, J. W. Jeong, S. H. Kim and K. W. Kim, "Hypoxia-Inducible Factor (HIF-1) Alpha: Its Protein Stability and Biological Functions,” Experimental \& Molecular Medicine, Vol. 36, No. 1, 2004, pp. 1-12. http://dx.doi.org/10.1038/emm.2004.1

[9] N. Ferrara, H. P. Gerber and J. LeCouter, “The Biology of VEGF and Its Receptors,” Nature Medicine, Vol. 9, No. 6, 2003, pp. 669-676. http://dx.doi.org/10.1038/nm0603-669

[10] J. H. Kim, M. Y. Oh, J. Paek and J. Lee, “Association between FGFR1OP2/wit3.0 Polymorphisms and Residual Ridge Resorption of Mandible in Korean Population,” PLoS ONE, Vol. 7, No. 8, 2012, Article ID: e42734.

[11] M. S. Rogers and R. J. D’Amato, “The Effect of Genetic Diversity on Angiogenesis," Experimental Cell Research, Vol. 312, No. 5, 2006, pp. 561-574. http://dx.doi.org/10.1016/j.yexcr.2005.10.021

[12] M. GarcÃa-Closas, N. Malats, F. X. Real, M. Yeager, R. Welch, D. Silverman, et al., "Large-Scale Evaluation of Candidate Genes Identifies Associations between VEGF Polymorphisms and Bladder Cancer Risk," PLOS Genetics, Vol. 3, No. 2, 2007, Article ID: e29. 